\title{
Generalized Perona-Malik Equation for Image Restoration
}

\author{
Guo W. Wei, Member, IEEE
}

\begin{abstract}
This letter introduces generalizations of the Perona-Malik equation. An edge enhancing functional is proposed for direct edge enhancement. A number of super diffusion operators is introduced for fast and effective smoothing. Statistical information is utilized for robust edge-stopping. Numerical integration is conducted by using a recently developed quasiinterpolating wavelet method. Computer experiments indicate that the present algorithm is very efficient for edge-detecting and noise-removing.
\end{abstract}

\section{INTRODUCTION}

$\mathbf{T}$ HE PERONA-MALIK equation [1], proposed in 1990, has recently stimulated a great deal of interest in image processing community [2]-[12]. It is commonly believed that the Perona-Malik equation provides a potential algorithm for image segmentation, noise removing, edge detection, and image enhancement. The basic idea behind the Perona-Malik algorithm is to evolve an original image, $I(\mathbf{r})$, under an edgecontrolled diffusion operator [1]

$$
\begin{aligned}
\frac{\partial u(\mathbf{r}, t)}{\partial t} & =\nabla \cdot[d(|\nabla u(\mathbf{r}, t)|) \nabla u(\mathbf{r}, t)] \\
u(\mathbf{r}, 0) & =I(\mathbf{r}) .
\end{aligned}
$$

Here, $d(|\nabla u|)$ is generalized diffusion coefficient which is so designed that its values are very small at the edge of an image. Perona and Malik argued that their anisotropic diffusion equation has no additional maxima (minima) which do not belong to the initial image data. This point has been challenged recently [2], [10]. It is well-known [2], [3], [5], [6] that this anisotropic diffusion algorithm may break down when the gradient generated by noise is comparable to image edges and features. However, this can be alleviated by using a regularization procedure.

The purpose of this letter is to introduce generalizations of the Perona-Malik equation. We introduce an edge-controlled enhancing functional for image enhancement. Super diffusion operators are introduced for fast and effective smoothing. Statistical information is used for robust edge-stopping. A recently developed quasi interpolating wavelet algorithm [13] is utilized for the numerical integration of generalized PeronaMalik equations. The efficiency and robustness of the present

Manuscript received December 17, 1998. This work was supported in part by the National University of Singapore. The associate editor coordinating the review of this manuscript and approving it for publication was Prof. S. Reeves.

The author is with the Department of Computational Science, National University of Singapore, Singapore 119260, Republic of Singapore (e-mail: cscweigw@nus.edu.sg).

Publisher Item Identifier S 1070-9908(99)04962-7. approach is illustrated by the restoration and enhancement of a noisy Lena image.

\section{THEORY}

In many cases, edges of an image may be worn down for various reasons. In other cases, the resolution of an image can be very low. Hence, a direct feature enhancement is often required. We propose a real-valued, bounded edge enhancing functional

$$
e(u(\mathbf{r}, t),|\nabla u(\mathbf{r}, t)|) .
$$

Here $e(u(\mathrm{r}, t),|\nabla u(\mathbf{r}, t)|)$ is appropriately chosen so that it is edge sensitive and the contrast of image edges is enhanced. This leads to a generalized Perona-Malik equation

$$
\begin{aligned}
\frac{\partial u(\mathbf{r}, t)}{\partial t}= & \nabla \cdot[d(u(\mathbf{r}, t),|\nabla u(\mathbf{r}, t)|) \nabla u(\mathbf{r}, t)] \\
& +e(u(\mathbf{r}, t),|\nabla u(\mathbf{r}, t)|) \\
u(\mathbf{r}, 0)= & I(\mathbf{r}) .
\end{aligned}
$$

Obviously, for constant $d$ and a simple form of $e$, (3) reduces to the inhomogeneous diffusion equation.

The heat equation can be derived from Fourier's law for heat flux, $\mathbf{j}_{1}(\mathbf{r}, t)=-D_{1} \nabla u(\mathbf{r}, t)$, with $D_{1}$ being a constant. This, from the point of view of kinetic theory, is an approximation to a quasi-homogeneous system that is near equilibrium. A better approximation can be expressed as a super flux

$$
\mathbf{j}_{\mathbf{q}}(\mathbf{r}, t)=-\sum_{q} D_{q} \nabla \nabla^{2 q} u(\mathbf{r}, t)
$$

where $D_{q}$ are constants and high order terms $(q>1)$ describe the influence of inhomogeneity in temperature field and fluxflux correlations to the heat flux. In particular, $\nabla \nabla^{2}$ can be regarded as an energy flux operator. Energy conservation leads to

$$
\begin{aligned}
\frac{\partial u(\mathbf{r}, t)}{\partial t} & =-\nabla \cdot \mathbf{j}_{\mathbf{q}}(\mathbf{r}, t)+s(\mathbf{r}, t) \\
& =\sum_{q} \nabla \cdot\left[D_{q} \nabla \nabla^{2 q} u(\mathbf{r}, t)\right]+s(\mathbf{r}, t)
\end{aligned}
$$

where $s$ is a source term which can be a nonlinear function. Equation (5) is a generalized reaction-diffusion equation which includes not only the usual diffusion and production terms, but also super diffusion terms. The case of second order super flux, $\mathbf{j}_{2}(\mathbf{r}, t)=-D_{1} \nabla u(\mathbf{r}, t)+\left|D_{2}\right| \nabla \nabla^{2} u(\mathbf{r}, t)$, has been used for the decription of a number of physical phenomena, such as pattern formation in alloys, glasses, polymer, combustion, and biological systems. 


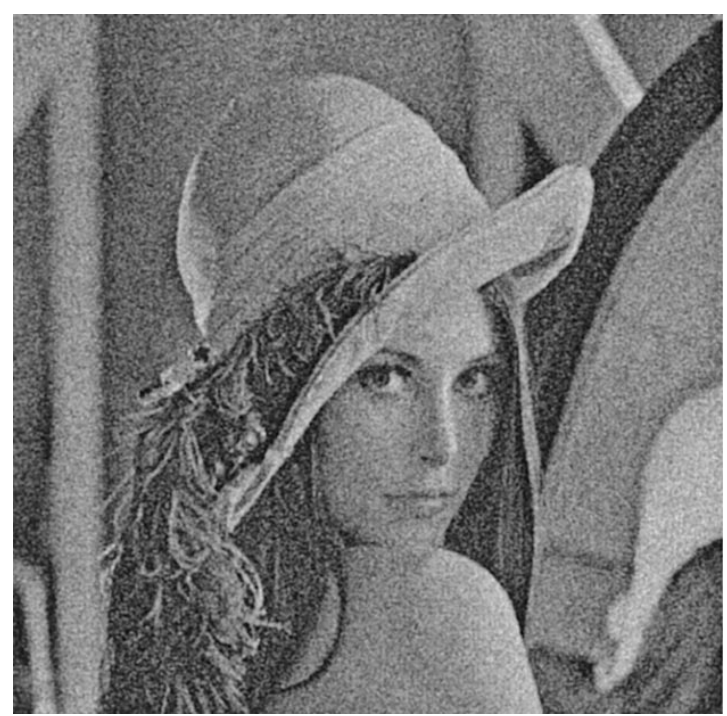

(a)

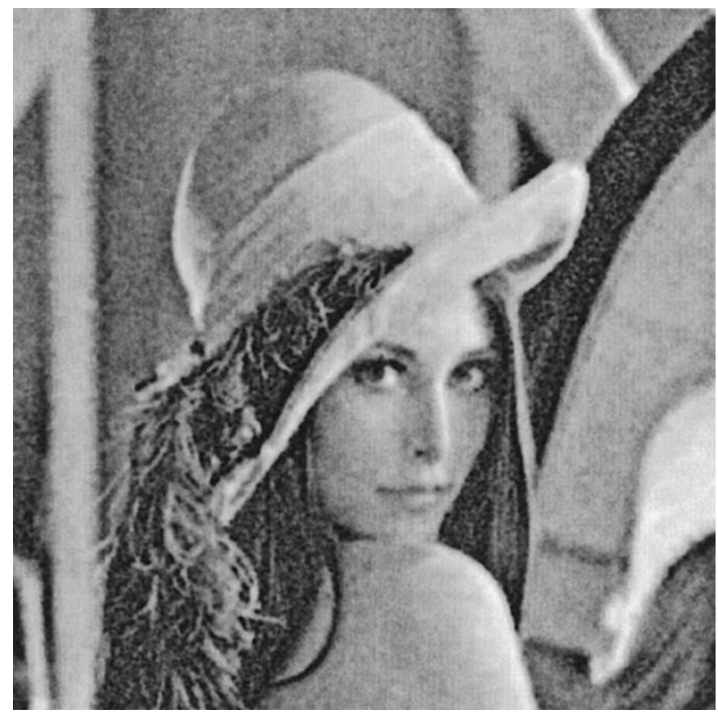

(c)

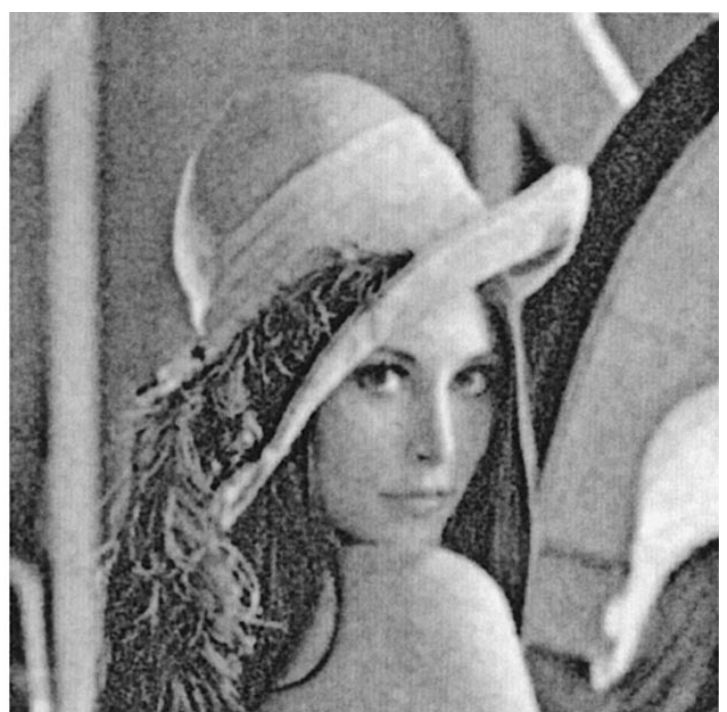

(b)

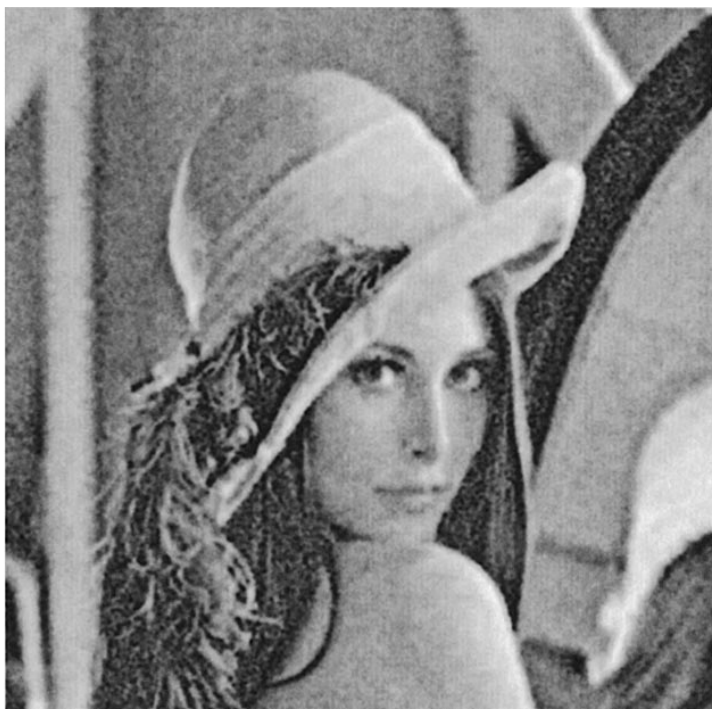

(d)

Fig. 1. Restoration of a noisy Lena image. (a) Noisy Lena image. (b) Restored with the Perona-Malik diffusion operator. (c) Restored with the Perona-Malik diffusion operator and the edge enhancing functional. (d) Restored with the Perona-Malik diffusion operator, the super-diffusion operator, and the edge enhancing functional.

In image systems, the distribution of image pixels can be highly inhomogeneous. Hence the generalized Perona-Malik equation (3) can be made more efficient for image segmentation and noise removing by incorporating an edge sensitive super diffusion operator

$$
\begin{aligned}
\frac{\partial u(\mathbf{r}, t)}{\partial t}= & \sum_{q} \nabla \cdot\left[d_{q}(u(\mathbf{r}, t),|\nabla u(\mathbf{r}, t)|) \nabla \nabla^{2 q} u(\mathbf{r}, t)\right] \\
& +e(u(\mathbf{r}, t),|\nabla u(\mathbf{r}, t)|) .
\end{aligned}
$$

Here $d_{q}(u,|\nabla u|)$ are edge sensitive diffusion functions. A special form used in this Letter for numerical experiments is

$$
\begin{aligned}
\frac{\partial u(\mathbf{r}, t)}{\partial t}= & \nabla \cdot\left[d_{1}(u(\mathbf{r}, t),|\nabla u(\mathbf{r}, t)|) \nabla u(\mathbf{r}, t)\right] \\
& +\nabla \cdot\left[d_{2}(u(\mathbf{r}, t),|\nabla u(\mathbf{r}, t)|) \nabla \nabla^{2} u(\mathbf{r}, t)\right] \\
& +e(u(\mathbf{r}, t),|\nabla u(\mathbf{r}, t)|) .
\end{aligned}
$$

The diffusion functions can be appropriately chosen in many different ways. In this letter, we choose the Gaussian for both $d_{1}(u(\mathrm{r}, t),|\nabla u(\mathrm{r}, t)|)$ and $d_{2}(u(\mathbf{r}, t),|\nabla u(\mathrm{r}, t)|)$

$$
d_{q}(u,|\nabla u|)=d_{q 0} \exp \left[-\frac{|\nabla u|^{2}}{2 \sigma_{1}^{2}}\right]
$$

where $d_{q 0}$ is automatically determined by the noise level, $\sigma_{0}$. Here both $\sigma_{0}$ and $\sigma_{1}$ are chosen as a local statistical variance of $u$ and $\nabla u$

$$
\sigma_{q}^{2}(\mathbf{r}, t)=\overline{\left|\nabla^{q} u-\overline{\nabla^{q} u}\right|^{2}}, \quad(q=0,1)
$$

where $\overline{X(\mathbf{r})}$ denotes the local average of $X(\mathbf{r})$ centered at point $\mathbf{r}$. The area of local average used in this work is $17 \times 17$ pixel $^{2}$. In this work, we simply choose the edge enhancing functional as

$$
e(u,|\nabla u|)=e_{0}(u) \frac{|\nabla u|}{\sigma_{1}\left(t+t_{0}\right)}
$$

with $e_{0}(u)$ varying as a function of $u(\mathbf{r}, t)$ and $\sigma_{0}(\mathbf{r}, t)$. 


\section{Numerical AsPeCtS AND Results}

The Perona-Malik equation (1) and generalized Perona-Malik equations (3) and (7) are spatially discretized using a quasi-interpolating wavelet algorithm [13]

$$
\begin{aligned}
\frac{\partial^{l} u(x, y)}{\partial x^{m} \partial y^{l-m}}= & \sum_{k=-W_{x}}^{W_{x}} \sum_{j=-W_{y}}^{W_{y}} \bar{\Phi}_{\sigma_{x}, \Delta_{x}}^{(m)}\left(x-x_{k}\right) \\
& \times \bar{\Phi}_{\sigma_{y}, \Delta_{y}}^{(l-m)}\left(y-y_{j}\right) u\left(x_{k}, y_{j}\right)
\end{aligned}
$$

where $\bar{\Phi}_{\sigma_{y}, \Delta_{y}}^{(m)}\left(x-x_{k}\right)$ is the $m$ th derivative of the quasi interpolating wavelet scaling function [13]. Here we choose $\sigma_{x} / \Delta_{x}=\sigma_{y} / \Delta_{y}=3.2$ and $W_{x}=W_{y}=8$ for removing noise and accurately detecting edges. Since our quasiinterpolating wavelet algorithm has a Schwartz class kernel, no additional regularization procedure is needed. Reflecting boundary condition is used in the time integration. The fourth order Runge-Kutta method is employed for the time discretization of the Perona-Malik equation and its generalizations. A total of 50 iterations is used in all integrations.

We use a $512 \times 512$ Lena image for our demonstration. The Lena image is degraded with Gaussian noise to obtain a peak signal-to-noise ratio (PSNR) of $25 \mathrm{~dB}$ [Fig. 1(a)]. The PSNR used here is calculated as

$$
\mathrm{PSNR}=\frac{255 \times 255}{\frac{1}{N_{x} N_{y}} \sum_{i=1}^{N_{x}} \sum_{j=1}^{N_{y}}[I(i, j)-u(i, j)]^{2}}
$$

where $I(i, j)$ and $u(i, j)$ are the original image and noisy image samples, respectively, and $N_{x}$ and $N_{y}$ are the number of pixels horizontally and vertically, respectively. A threshold value of 38 is used. Fig. 1(b) shows the restored Lena images produced by using the Perona-Malik equation (1). The increase in PSNR is quite large $(22 \mathrm{~dB})$ with the use of the auto threshold technique and the quasi-interpolating wavelet algorithm. Fig. 1(c) is obtained by the combined use of the edge enhancing functional and the Perona-Malik diffusion operator (3). Obviously, the features of the Lena image are effectively enhanced by the edge enhancing functional as indicated by an increase of PSNR of $33 \mathrm{~dB}$. The performance of the full generalized Perona-Malik equation (7), is given in Fig. 1(d) (PSNR $=71 \mathrm{~dB})$. It is clearly seen that the present algorithm can effectively remove noise while preserving the edges simultaneously. We have also tested our algorithm for nosier images and have found that it can increase the PSNR enormously.

\section{CONCLUSION}

This letter presents generalized Perona-Malik equations for edge-detected image processing. An edge enhancing functional is introduced for direct image enhancement. Such a functional is very useful for treating low contrast images and edge blurred images. We also introduce edge-detected super diffusion operators for highly inhomogeneous images. The super diffusion operators are very efficient for noiseremoving. Statistical information is used for the automatical choice of edge-stopping threshold. A newly developed quasiinterpolating wavelet technique is used for the time integration of nonlinear partial differential equation systems. Numerical experiments with a noisy Lena image show that the generalized Perona-Malik equations are effective for noise-removing and simultaneously edge-preserving.

\section{ACKNOWLEDGMENT}

The author thanks Dr. E. C. Chang, Dr. Y. P. Wang, and Prof. Y. Yi for useful discussions.

\section{REFERENCES}

[1] P. Perona and J. Malik, "Scale-space and edge detection using anisotropic diffusion," IEEE Trans. Pattern Anal. Machine Intell., vol. 12, pp. 629-639, 1990.

[2] F. Catte, P.-L. Lions, J.-M. Morel, and T. Coll, "Image selective smoothing and edge detection by nonlinear diffusion," SIAM J. Numer. Anal., vol. 29, pp. 182-193, 1992.

[3] M. Nitzberg and T. Shiota, "Nonlinear image filtering with edge and corner enhancement," IEEE Trans. Pattern Anal. Machine Intell., vol. 14, pp. 826-833, 1992.

[4] L. Rudin, S. Osher, and E. Fatemi, "Nonlinear total variation based noise removal algorithm," Physica D, vol. 60, pp. 259-268, 1992.

[5] R. T. Whitaker and S. M. Pizer, "A multi-scale approach to nonuniform diffusion," CVGIP: Image Understand., vol. 57, pp. 99-110, 1993.

[6] Y.-L. You, W. Xu, A. Tannenbaum, and M. Kaveh, "Behavior analysis of anisotropic diffusion in image processing," IEEE Trans. Image Processing, vol. 5, pp. 1539-1553, 1996.

[7] F. Torkamani-Azar and K. E. Tait, "Image recovery using the anisotropic diffusion equation," IEEE Trans. Image Processing, vol. 5, pp. 1573-1578, 1996.

[8] J. Shah, "A common framework for curve evolution, segmentation and anisotropic diffusion," in Proc. IEEE Conf. Computer Vision and Pattern Recognition, San Francisco, CA, June 1996, pp. 136-142.

[9] S. T. Acton, "Edge enhancement of infrared imagery by way of anisotropic diffusion pyramid," in Proc. IEEE 3rd Int. Conf. Image Processing, Lausanne, Switzerland, Sept. 1996, pp. 865-868.

[10] M. Kichenassamy, "The Perona-Malik paradox," SIAM J. Appl. Math., vol. 57, pp. 1328-1342, 1997.

[11] S. Teboul, L. Blanc-Feraud, G. Aubert, and M. Barlaud, "Variational approach for edge-preserving regularization using coupled PDE's," IEEE Trans. Image Processing, vol. 7, pp. 387-397, 1998.

[12] M. J. Black, G. Sapiro, D. H. Marimont, and D. Heeger, "Robust anisotropic diffusion," IEEE Trans. Image Processing, vol. 7, pp. 421-432, 1998

[13] G. W. Wei, "Quasi wavelets and quasi interpolating wavelets," Chem. Phys. Lett., vol. 296, pp. 215-222, 1998. 